\title{
ScienceRobotics
}

\section{New soft robots really suck: vacuum powered systems empower diverse capabilities}

\author{
M. A. Robertson, ${ }^{1}$ J. Paik ${ }^{1 *}$ \\ ${ }^{1}$ Reconfigurable Robotics Laboratory, École Polytechnique Fédérale de Lausanne (EPFL), 1015 Lausanne, \\ Switzerland. \\ *Corresponding author. Email: jamie.paik@epfl.ch
}

\begin{abstract}
We introduce a vacuum-powered soft pneumatic actuator (V-SPA) which leverages a single, shared vacuum power supply and enables complex soft robotic systems with multiple-DoF and diverse functions. In addition to actuation, other utilities enabled by vacuum pressure include gripping and stiffening through granular media jamming, as well as direct suction adhesion to smooth surfaces, for manipulation or vertical fixation. We investigate the performance of the new actuator through direct characterization of a 3-DoF, plug-and-play V-SPA Module built from multiple V-SPAs, and demonstrate the integration of different vacuum-enabled capabilities with a continuum-style robot platform outfitted with modular peripheral mechanisms. We show these different vacuum-powered modules can be combined to achieve a variety of tasks, including multi-modal locomotion, object manipulation, and stiffness tuning to illustrate the utility and viability of vacuum as a singular alternative power source for soft pneumatic robots, and not just a peripheral feature in itself. Our results highlight the effectiveness of V-SPAs in providing core soft robot capabilities and facilitating the consolidation of previously disparate subsystems for actuation and various specialized tasks, conducive to improving the compact design efficiency of larger, more complex multi-functional soft robotic systems.
\end{abstract}

\section{Summary}

We introduce a foam-based vacuum powered actuator and exemplary complex, multifunctional soft robotic system which it enables.

\section{Introduction}

Robots that operate in highly variable environments or in close cooperation with humans require both robustness and adaptability to ensure reliability and safety. These features can be accommodated most easily by adding compliance to a robotic system, which can be achieved either actively through impedance control methods $(1,2)$ or passively through compliant mechanical components and materials. In part, the advantage of a materialsbased approach to creating compliant systems is to offload some of the burden of complexity in control to morphological and material computation, in a sense, to achieve robust and adaptable behavior $(3,4)$. This latter strategy has been the recent focal point of the field of soft robotics, where inherently compliant and flexible materials such as silicone rubber are used to fabricate primary structural and active robot components (5). Various forms of soft pneumatic actuators (SPAs) powered by pneumatic pressure have been developed in this domain to compliment the nature of these soft materials, which stretch and bend through inflation or deformation of elastic chambers to produce useful mechanical work $(6-16)$. 
This relatively new approach to robotics has yielded mobile platforms, manipulators, and other soft structures which are applicable in increasingly complex applications. Rough terrain locomotion, delicate handling, and human interaction tasks all benefit from the natural quality of SPA driven systems to conform or yield safely to rigid environmental constraints without sacrificing the functional purpose of the device (17-19). While now traditional soft pneumatic systems afford these benefits already utilizing positive pressure, recent interest in negative pressure systems aims to improve these qualities further. Exploiting the effect of mechanical buckling to generate controlled force, vacuum driven soft, muscle-like actuators have been successfully demonstrated utilizing standard soft robotic materials and fabrication techniques $(20,21)$. This type of actuator offers implicitly fail-safe operation, being limited by environmental pressure from actuating beyond a maximum force and displacement, and directly enables contractile motion, more similar to biological muscle than the expansion-based motions of many positive pressure driven soft actuators.

Following previous effort $(20,21)$, we introduce a new vacuum-powered Soft Pneumatic Actuator, the V-SPA, to expand the diversity and utility of this versatile power source for new soft robots. In comparison to the few existing examples of vacuum driven actuators, this new type of actuator is exceptionally rapid to fabricate, for proficient iteration through multiple designs. This is a result of requiring no molds or sacrificial compounds and being constructed primarily of readily available, manufactured foam sheets. The use of porous foam structures in soft robotics has recently been investigated to a limited extent for various novel benefits. Employing self-manufactured poroelastic foam, a bioinspired fluid pump was developed to showcase the utility and simplicity of foam-based compliant actuation (22). Following a similar method to fabricate poroelastic foam from a moldable compound infused with a fugitive salt porogen, highly customized inflatable structures can be sculpted in free form for laboratory, art, or classroom educational purposes (23). Other work has explored the use of bicontinuous metal-elastomeric foam to achieve variable stiffness and self-healing properties (24). While most of these methods have been applied toward the fabrication of SPA devices powered by positive pressurized air, the application of vacuum to foam structures is an approach that combines the advantages of vacuum power, with some of those inherited from the properties of foam material itself, including impact resistance, robustness, and storage-friendly "crushability" (25). We introduce here a new type of soft actuator to exploit this combination, enabling a wide range of new soft robotic applications, including safe, lightweight, wearable devices, collaborative machines, or packable mobile robots that are space and energy efficient, suitable for remote deployment or even possibly flight (see Figure 1). 


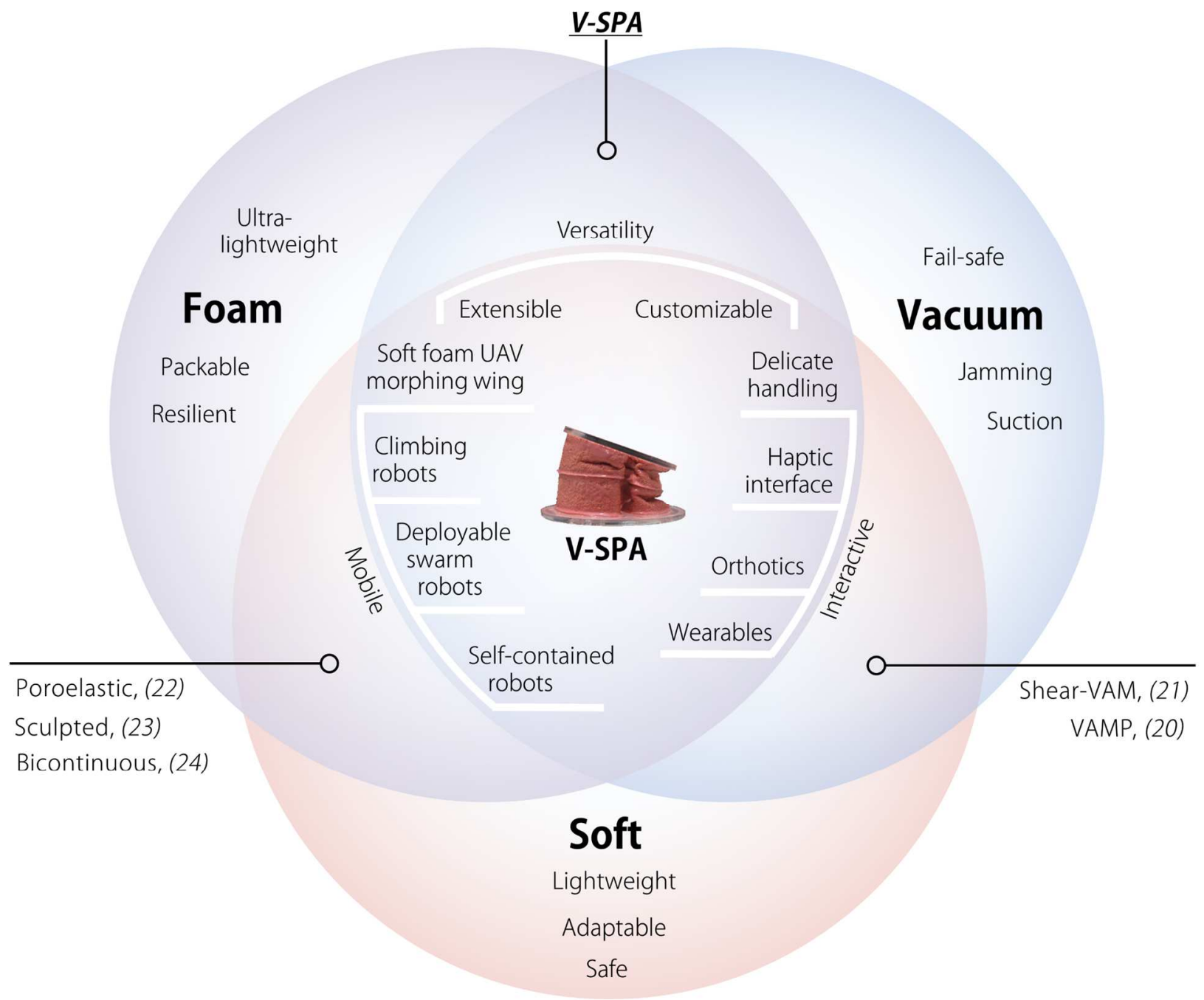

Fig. 1. V-SPAs blend multiple material and operational domains for diverse potential applications. Existing work independently explored the use of vacuum and foam materials respectively for the development of soft robotic systems, to leverage the unique attributes of robustness, safety, and manufacturability offered by different fabrication and actuation methods. This work explores a new approach which leverages foam and vacuum power simultaneously with an actuator called the V-SPA, to take advantage of a new construction technique which enables rapid production of soft robotic systems using minimal resources, as well as open possibility for fully vacuum powered systems which enable soft robots with expanded capabilities.

Capitalizing on the vacuum supply available for actuation, vacuum-based soft robotic systems can also leverage this source to incorporate other functions and features powered by negative pressure, without the need for an additional power supply or additional subsystem infrastructure. Vacuum powered mechanisms have been shown to enable operations useful to soft robots, including gripping (26), suction (27), and stiffness tuning (28-31). By adding to this variety of available vacuum driven mechanisms a new lightweight alternative for actuation, V-SPAs open the opportunity to expand the capability of soft robots while actually minimizing the size and complexity of their design and implementation. The seamless integration of these mechanisms through a common vacuum supply not only yields more efficient soft robot design but subsequently facilitates highorder soft pneumatic systems with many DoFs, which are still relatively scarce in the field of soft robotics. To demonstrate this facility, we employ the use of V-SPAs in a typically 
complex and difficult multi-DoF robot morphology, generally referred to as a continuum robot. Also known as Hyper-Redundant Robot Manipulators (HRRMs), trunks, tentacles, or snakes, these narrow structures often consist of a linear chain of repeating modular units, each with single or multiple active DoF. This concept enables a high degree of kinematic maneuverability and flexibility for either manipulation $(32-36)$, positioning $(31,37)$, or locomotion (38-40) tasks, to account for variable environments or system objectives. While the low-mass of the V-SPAs themselves make such an architecture with many DoF more feasible, dealing with the practical difficulty imposed by cumbersome, overlapping pneumatic supply lines routed from a central control source to many independent actuator units often limits its realistic execution. To overcome this barrier as well as allow the consolidation of multiple vacuum-based mechanisms into the same platform through a common vacuum supply line, we employed a decentralized, modular design approach.

This work validates the integration of our new actuator in multifunctional vacuum-driven soft robotic systems by experimental demonstration of interaction task versatility and mechanical performance tuning of physical properties through the use of plug-and-play control and design methods. By introducing new actuation technology and demonstrating the combination of different functions made possible through a negative pressure pneumatic power supply, we help to establish vacuum-power as a substantial alternative class of soft robotics that offers unique and important potential for realizing complex and advanced systems.

\section{Results}

\section{Vacuum-powered Soft Pneumatic Actuator (V-SPA)}

We designed and developed a critical robotic component, the V-SPA, which is characterized by its implicit reliability and safety through the utilization of vacuum power and the robust properties of a foam core. This new actuator is simple in design, and easy to fabricate without the need for either an internal or external mold. It is composed of a laser-cut, offthe-shelf foam core and thin, brushed-on layers of silicone rubber, and can be manufactured from scratch, ready to use in less than two hours. The foam core acts as an internal scaffold over which uncured silicone rubber can be applied to form a thin, sealing layer around the open-celled foam as it can be seen in Figure S1. This creates an enclosed, airtight structure which is only sparsely filled with soft, porous material. While conventional SPAs or other vacuum actuator designs feature completely hollow inner cavities, the use of foam allows the wall thickness of V-SPAs to be much thinner as it provides much of the structural support typically given by a thicker wall needed to maintain a nominal actuator shape. This difference in material wall thickness helps to offset the added mass of the core, yielding an ultimately lighter weight actuator overall. Upon activation, vacuum is applied to the internal volume through a supply channel and the entire V-SPA structure collapses inward to produce tensile force that can be used for actuation. The low-density core is easily deformed and crushed when vacuum is applied, but immediately provides elastic return in conjunction with the elasticity of the outer silicone membrane following actuation to return the actuator to its original shape. The activation of a circular V-SPA array can be seen in Movie S1.

In soft robotics, foam-based vacuum-powered actuators offer a solution to improve the efficiency of soft systems, by reducing mass in actuators, allowing for the integration of additional mass components onboard to enable fully self-contained systems, including battery or pressure (or direct vacuum) generating pumps. This reduction in the total system mass budget also makes room for larger systems with more DoF, enabling further complex 
behavior. While vacuum power is not suited for every application, in part due to limited force production at atmospheric pressure, for use at small scales where this force limit is perhaps beyond maximum requirements, and where this limit offers a unique safety feature as it prevents actuating beyond the force imposed by external pressure, V-SPAs hold a unique advantage at significantly low cost in weight, materials, and production effort.

\section{Scalable, reconfigurable soft robot}

We developed a robotic platform using V-SPAs to power hybrid (41) modular units, known as V-SPA Modules, which contain both soft actuators and rigid components necessary for their power and control. This architecture was chosen to practically implement V-SPAs in an example soft robotic system with enhanced and diverse capabilities. The modular design of the units enables rapid, simplified restructuring and control of various robot configurations for easy testing and validation of vacuum system extensibility. Specifically, these modules allow the addition or subtraction of mechanical DoF with little or no physical design effort through standardized pneumatic and electrical network connection ports. Other peripheral device modules with different functionalities can also be easily combined with the actuators in the same way. To eliminate the effort of developing customized control for each unique combination of modules in a scalable or multifunctional system, we employ a standard control scheme that does not change with the number or type of modules connected. While high level control planning and programming is still required to achieve specific tasks, the low-level management of multi-module integration is automatically taken care of through this flexible plug-and-play network architecture utilizing embedded control circuitry and hardware. Following this design approach, the resulting reconfigurable soft robotic system we produced embodies a typically challenging morphology, a hyperredundant continuum type robot not easily reproduced by other existing soft robot design methods.

\section{V-SPA Module configuration}

We produced V-SPA Modules using three actuators each, evenly spaced in a cylindrical configuration, and connected at the top and bottom to rigid plates which house or mount electronics, valves, pneumatic fittings, electrical connectors, and distribution channels connecting to a centralized vacuum supply line. Other work has leveraged this approach for more efficient configuration of mobile snake-like robots $(42,43)$, and use in a surgical continuum robot comprised of a serial chain of 3 -DoF soft actuator modules with miniature control valves embedded locally (44). Our V-SPA Modules similarly exploit the advantages of decentralized hardware architecture in order to reduce complexity, space, and weight of vacuum powered systems with integrated peripheral functions.

The three V-SPAs comprising each module are used to tilt the orientation of a rigid upper plate (conventional printed circuit board, PCB) relative to one at the bottom. The actuators are attached to these end plates and contract when activated, leading to angular deflection between them in a direction and degree defined by the combination of actuators activated simultaneously and the magnitude of their activation (by duty-cycle, proportional to length). Using a purely binary scheme to control the actuators fully on or off, the module actuators can be triggered independently or in pairs to produce six unique directions of motion at a fixed angle of deflection. The co-activation of all three actuators does not produce significant linear deflection as implemented here with a stiff supply conduit through the center, but this motion can easily be achieved without it. The motion of a V-SPA Module 
following this simple control method can be seen in Movie S2. More complex activation of the actuators was not shown here, but can be accomplished using PWM control for each actuator to achieve variable contraction, expanding the workspace to continuous, $360^{\circ}$ directed bending with variable angle. While the angular deflection of the module can be represented as combined rotations along two axes in space, we express this in terms of active DoF rather than spatial, to define each V-SPA Module as a 3-DoF actuated joint. Characterization testing of the V-SPA Modules presented in Figure 2 was performed by measuring the angular step response of a module under varied conditions. From the different response profiles, metrics were obtained relating actuator and module performance to different loading and supply pressure conditions. The measured characteristics are summarized in Table 1.

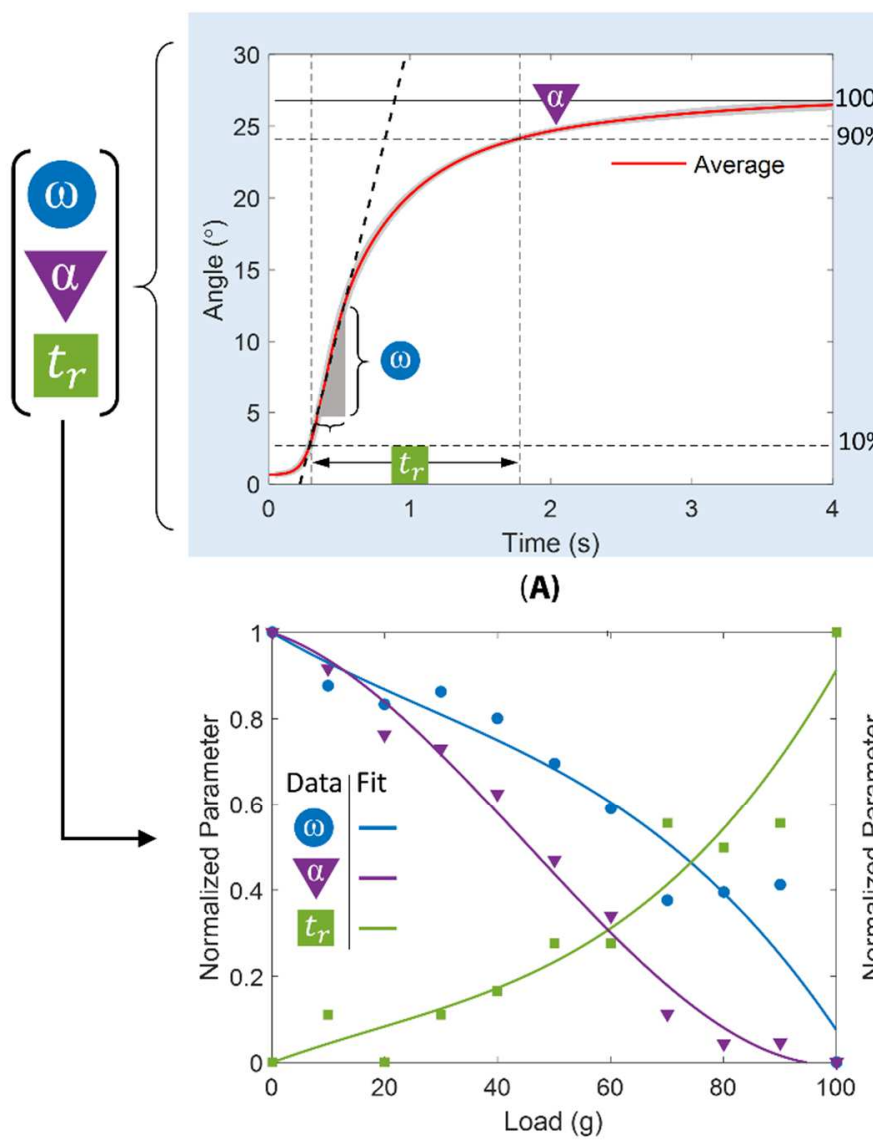

(C)

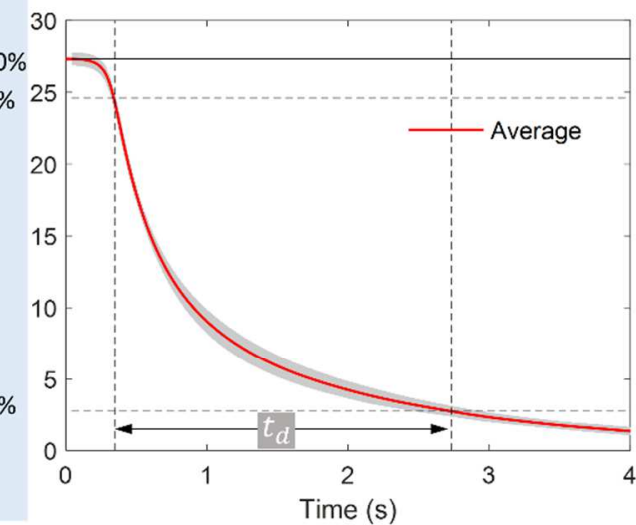

(B)

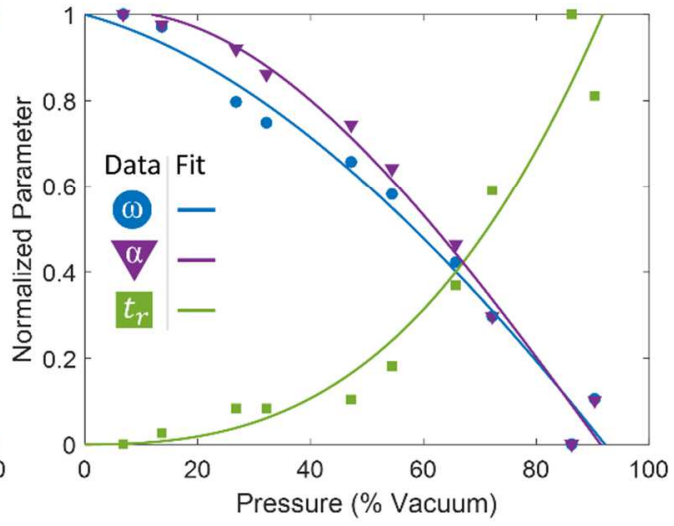

(D)

Fig. 2. V-SPA Module step response characterization. Three V-SPAs were characterized with a step input while measuring the angular response with an IMU. The average (red curve) step response at maximum vacuum with no load are shown in (A), and the step down profile is shown in (B). A single standard deviation is also shown in light grey shading, for 30 cycles ( 10 cycles for each of 3 actuators) comprising the average response. The metrics found from the average step response for variable loads are shown in (C) and variable pressure in (D), and the trends plotted with a third order polynomial fit.

Table 1. Physical properties and performance of V-SPA Module. Performance metrics were estimated from an angular displacement step response test recorded relative to gravity using an IMU fixed to the upper stage of the module, and using $86.2 \%$ vacuum supply.

\begin{tabular}{lll} 
Properties & Value & Unit \\
\hline Size $(\mathrm{D} \times \mathrm{H})$ & $45 \times 45$ & $(\mathrm{~mm})$
\end{tabular}




$\begin{array}{lll}\text { Total module weight } & 45.0 & (\mathrm{~g}) \\ \text { V-SPAs (x3) } & 11.1 & (\mathrm{~g}) \\ \text { Solenoid valves (x3) } & 12.0 & (\mathrm{~g}) \\ \text { PCBs } & 8.5 & (\mathrm{~g}) \\ \text { Acrylic and epoxy layers } & 8.5 & (\mathrm{~g}) \\ \quad \text { Central conduit (tubes, connectors, wires) } & 4.9 & (\mathrm{~g}) \\ \text { Blocked torque } & 166.9 & (\mathrm{~N} \cdot \mathrm{mm}) \\ \text { Specific torque (rel. to Actuator mass) } & 45.3 & (\mathrm{~N} \cdot \mathrm{mm} / \mathrm{g}) \\ \text { Angular velocity, } \omega \text { (no load) } & 3.5 & (\% / \mathrm{s}) \\ \text { Step rise time, } t_{r} & 1.5 & (\mathrm{~s}) \\ \text { Step decay time, } t_{d} & 2.4 & (\mathrm{~s}) \\ \text { Bandwidth } & 0.2 & (\mathrm{~Hz}) \\ \text { Max. angular stroke, } \alpha \text { (no load) } & 27.3 & \left({ }^{\circ}\right) \\ \text { Specific power (rel. to Actuator mass) } & 5.0 & (\mathrm{~W} / \mathrm{kg}) \\ \text { Specific energy (rel. to Actuator mass) } & 1.2 & (\mathrm{~J} / \mathrm{kg})\end{array}$

Each V-SPA Module connects in series to another through connections on the top and bottom to a central pneumatic supply line, power and ground lines, and communication bus, shown in Figure 3A. The overall structure of the resulting network of modules can be seen in Figure 3B with the note that this architecture is not limited to efficiently integrating actuators alone but is designed to facilitate rapid and simple extension of many different module types for vacuum (or other) pneumatic robotic systems. Commands to individual actuators in each networked module are relayed over a communication signal line using a robust serial protocol designed initially for low-cost RGB LED serial displays. Since each channel of every module in the network is identified sequentially and not through a unique address, this allows modules to be added or subtracted in a generic way that does not affect the low-level control programming. While such modules could be used in various combinations, in a multitude of different robot topologies, the nominal configuration studied here in direct linear arrangement allowed for the demonstration of many interesting features enabled by V-SPAs and vacuum power alike. 


\section{Exploded view of V-SPA Module}
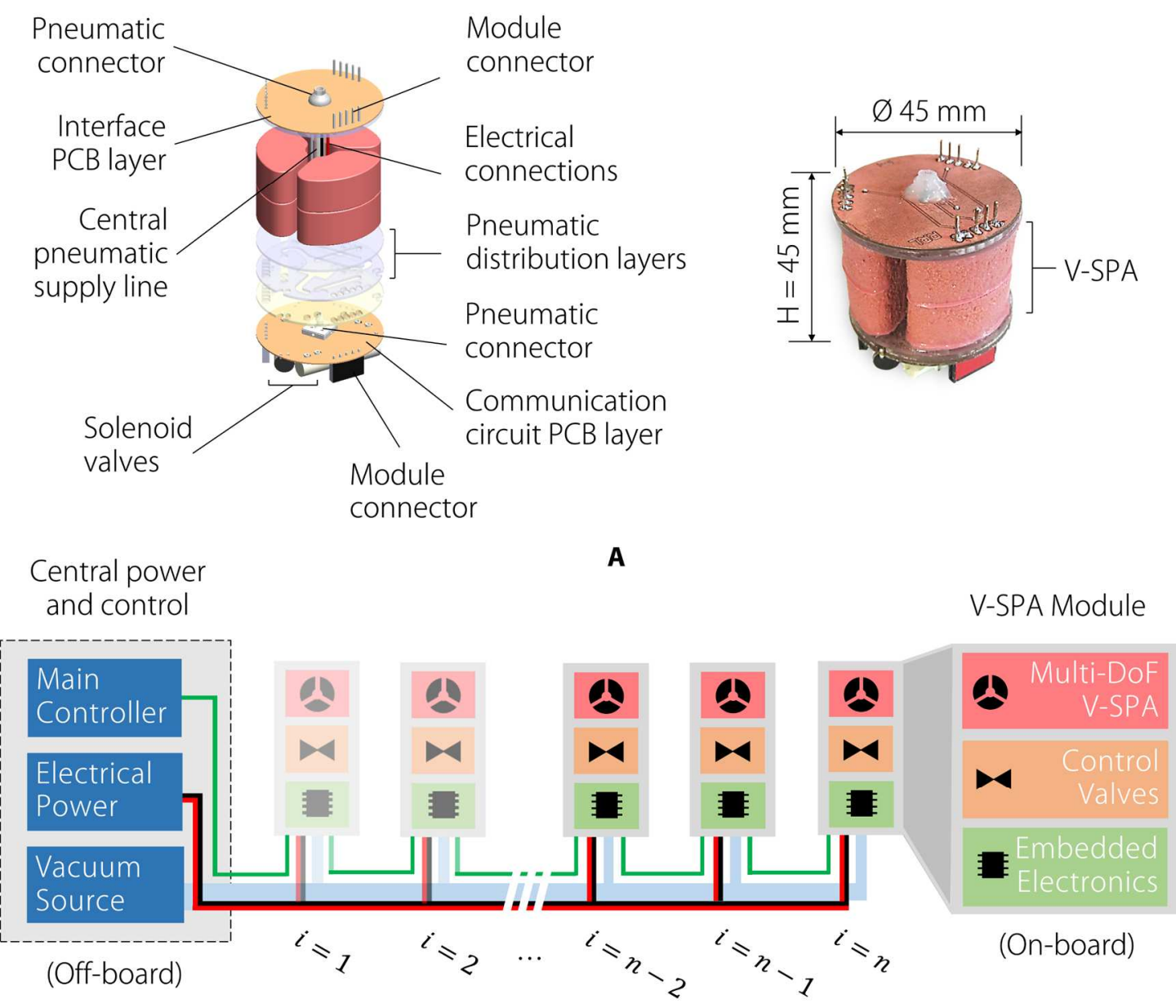

B

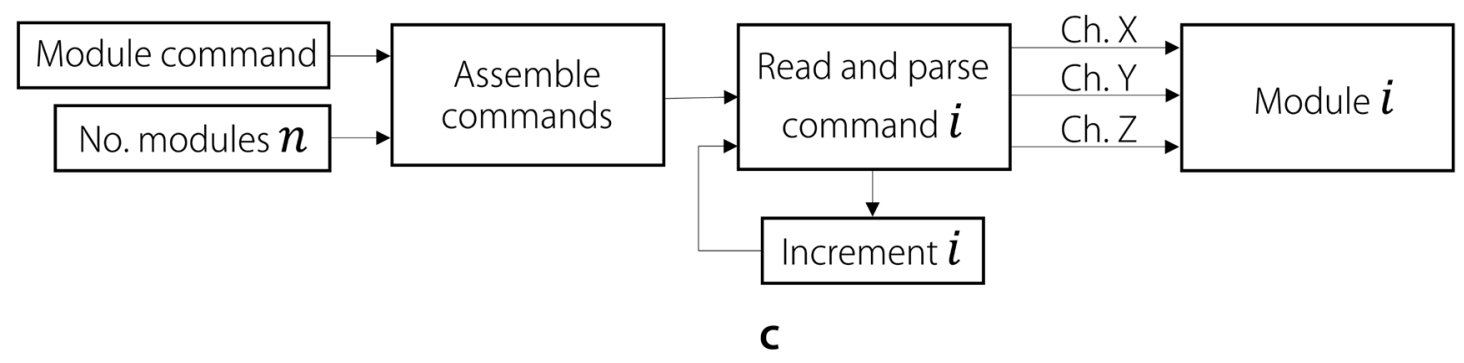

Fig. 3. Architecture of V-SPA Module and integrated soft modular device network. (A) Each module contains three main components: actuators (V-SPA), control valves, and electronics. (B) Each actuator in the module is paired to a valve, and dedicated channel of a communication IC. A common pneumatic supply line provides fluid power to every module simultaneously, while a common electrical bus similarly provides electrical power. (C) Control commands are assembled into data packets by a main controller, and relayed by the IC through each module connected in series. Each module has three independent channels $(\mathrm{X}, \mathrm{Y}$, and Z) which can be addressed to control up to three embedded valves in open-loop. 


\section{Hyper-redundant soft robot performance}

We first tested the operation of a five module robot to quantify its performance in the simplest form, as a soft manipulator arm. The workspace and repeatability of the robot's three principle directions of motion from an initially vertical standing orientation were measured using an external OptiTrack motion capture system in order to demonstrate the practical utility of a V-SPA based system. The proximal end of the robot was fixed to a stationary base, while three markers were attached to the distal end to track the 3D trajectory of their centroid, treated as the robot endpoint. For this preliminary evaluation, every module in the robot was synchronized to move the endpoint virtual marker through three distinct locations (roughly $0^{\circ}, 120^{\circ}$, and $240^{\circ}$ ) representing the robots principle directions of motion as shown in Figure 4A. Binary activation signals (on/off) were used to achieve the maximum bend in each of these directions, although independent control of each of the 15 total V-SPAs in the robot (three per module) is possible, and continuous motion of each actuator or combination of actuators in a single module could also be achieved using PWM signals to produce motion in any arbitrary angular direction.

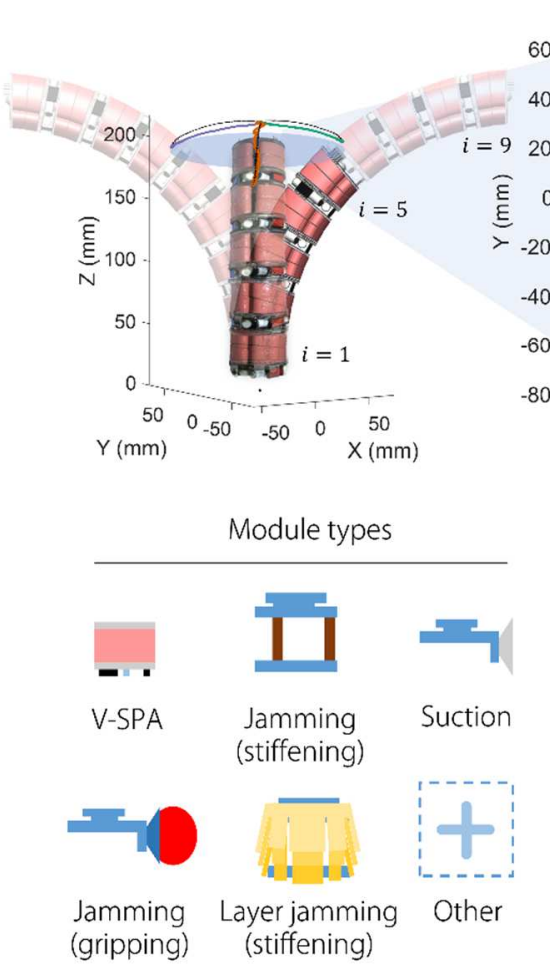

(B)

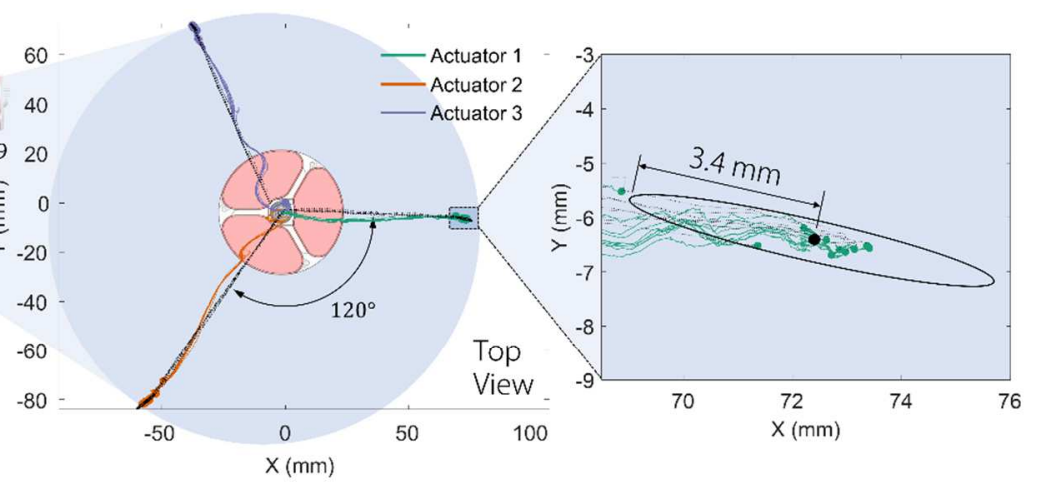

(A)

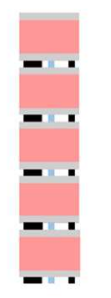

(3)

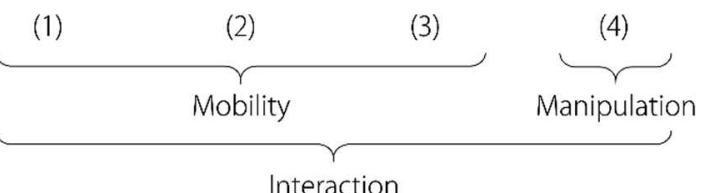

(C)

Fig. 4. Versatility of vacuum-powered soft hyper-redundant robot. The workspace and repeatability of 5module soft hyper-redundant robot is depicted in (A) along with a depiction of the maximum reach for up to 9 modules. To measure the range of motion along three primary directions determined by the actuators in each module, three markers were placed on the top of the distal module, and a centroid was calculated to track the center of the module as the robot endpoint. A top view of the distal module centroid 3D trajectory is shown through a ten cycle repeatability test. A variety of module types shown in (B) can be readily integrated with the hyper-redundant robot. The configurations in $(\mathbf{C})$ combining various modules are all validated experimentally in subsequent sections.

The robot endpoint position was measured for ten cycles through each location and a 2D error ellipse (95\% confidence interval) was calculated for each set of ten points in each of the three locations (see supplemental Movie $\mathbf{S 3}$ for video of a 10-cycle trial test). The length 
of the ellipse major axes, corresponding to the largest variability in endpoint positioning, was averaged for all three locations. For a five-module robot, the average error recorded across the three principle locations tested was found to be $\pm 3.4 \mathrm{~mm}$. This corresponds to an average repeatability measure of $\pm 0.68 \mathrm{~mm}$ accuracy per module. Individually, the range of accuracy across each of the three respective directions differed slightly as a result of variable construction and individual actuator performance, with the lowest variability in a particular direction (actuator 3 ) measured to be $\pm 1.9 \mathrm{~mm}$ for the robot as a whole, or \pm 0.38 $\mathrm{mm}$ per module along that axis. While the path and final position of the robot endpoint in each direction are seen to be repeatable, deviations from a purely linear path (from the top view) between the neutral start point and end points can also be seen. In part this is because the actuators are driven by the inherently unstable mode of buckling of the V-SPA outer rubber skin, made variable between actuators by non-uniformity in their construction and materials. Despite this variability from manual fabrication, however, these measurements validate the use of V-SPAs for highly repeatable tasks, critical for practical robotic systems.

\section{Validation of reconfigurable soft robot capabilities}

To complement the mechanical flexibility of the hyper-redundant robot, a variety of module types can be easily integrated with it to achieve design and task flexibility. Possibilities for vacuum driven systems include those shown in Figure 4B. Three of the modules depicted are demonstrated in this work, while future modules yet to be implemented are proposed to further illustrate the capacity and utility of vacuum based soft robot design. We validate the diverse functionalities facilitated by these modules through demonstration of various interaction tasks between the robot and its environment, and an example of controllable mechanical tuning of dynamic system properties implemented through a plug-and-play modular design paradigm. Through the use of peripheral modules, these abilities extend the diversity of applications for soft robots, including enhanced mobility and controllability enabled by the configurations depicted in Figure 4C (1-4), highlighting the efficacy and versatility of V-SPAs and vacuum-based soft robotic systems as a whole.

\section{Interaction task: Suction manipulation}

As a first test of the versatility of the vacuum power supply, a suction manipulator component was added to the end of the positioning arm, maintaining a modular design approach. A snap-fit 3D printed part was attached to the distal robot module to provide the structural basis of the suction module, including a modified off-the-shelf soft suction cup. The new peripheral module was connected to the shared vacuum power supply, and a custom networked control valve plugged in series to the output electrical interface of the distal actuator module to be controlled along the common control signal bus. A silicone tube connected the valve output to the internal volume of a soft suction cup through a hole at the top, enabling it to be actively depressurized or vented to the atmosphere for controllable attachment or detachment to objects. The soft continuum manipulator arm was then tested in a simple reaching and manipulation task of smooth acrylic containers to demonstrate the performance of combined actuation and suction manipulation. The multifunctional system was able to successfully "grasp" and relocate objects from a starting location to target bins, as shown in Movie S4. 


\section{Interaction task: Vertical window climbing}

Integrating lightweight actuation with other vacuum-based mechanisms enables capabilities beyond positioning and manipulation tasks. We demonstrate further advantages to combining these mechanisms by reconfiguring V-SPA Modules and suction cup modules into a soft robot capable of vertical climbing on smooth surfaces. Two vital requirements for this type of robot are readily accommodated by the actuator and architecture presented previously; to provide active motion for climbing and selective attachment and release of robot footholds while maintaining low enough weight to not hinder the effect of those capabilities. The low-mass foam-core V-SPAs are naturally well-suited to this application, and the modular, shared vacuum supply architecture of the complete soft robotic system enables the efficient integration of the active functions necessary for vertical climbing in a nearly self-contained soft robotic system that can scale surfaces such as glass windows. Using only an open-loop, fixed gait pattern controller and without optimizing timing parameters to find maximum speed, demonstrated gait was measured to be $2 \mathrm{~mm} / \mathrm{s}$, or 0.01 Body Lengths per second (BL/s). While the climbing robot we present here lacks an onboard electrical power supply and pneumatic vacuum generation source, we verified it is capable of carrying additional payload up to $70 \mathrm{~g}$, which may be useful for accommodating the remaining components to enable a more autonomous robot in future. Figure $\mathbf{5 A}$ shows the progression of the climbing robot, and Movie S5 shows testing with increasing external payload, with an evident corresponding reduction in climbing speed. Although this change in climbing performance can be attributed in part to variable actuator functionality in response to load, this can also be a factor of the robot design which included a passive degree of freedom at the lower foot needed for climbing and an offset of the payload mass resulting in a moment which pulled the robot away from the wall and reduced step size. 
(A)

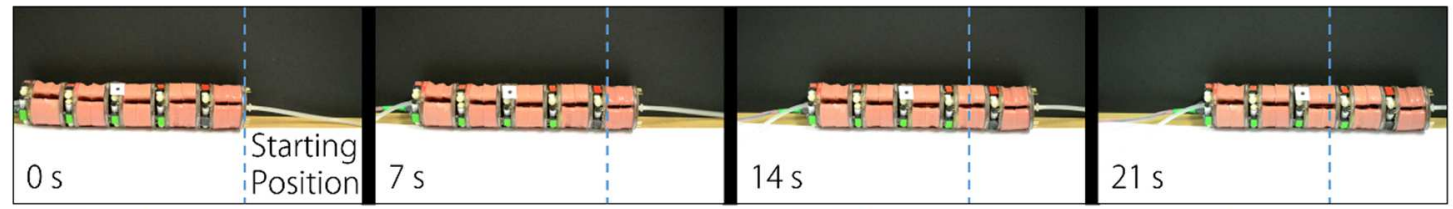

(B)

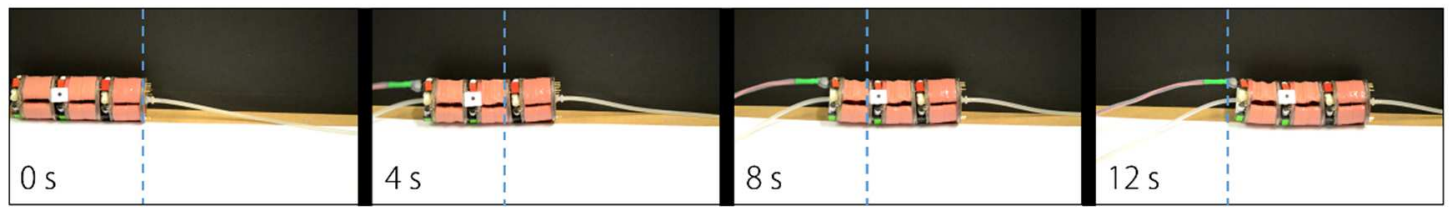

(C)

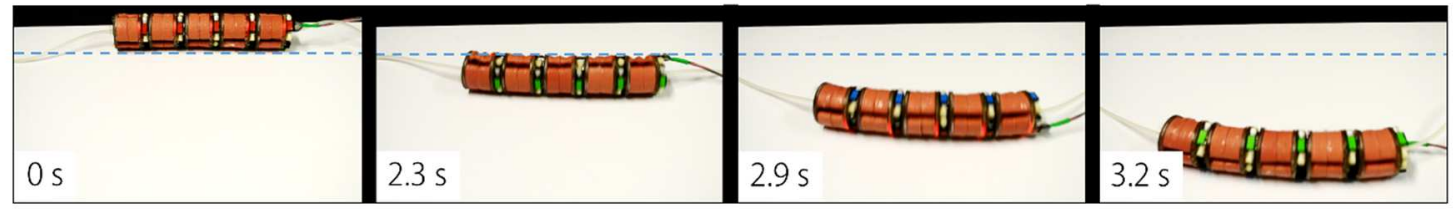

(D)

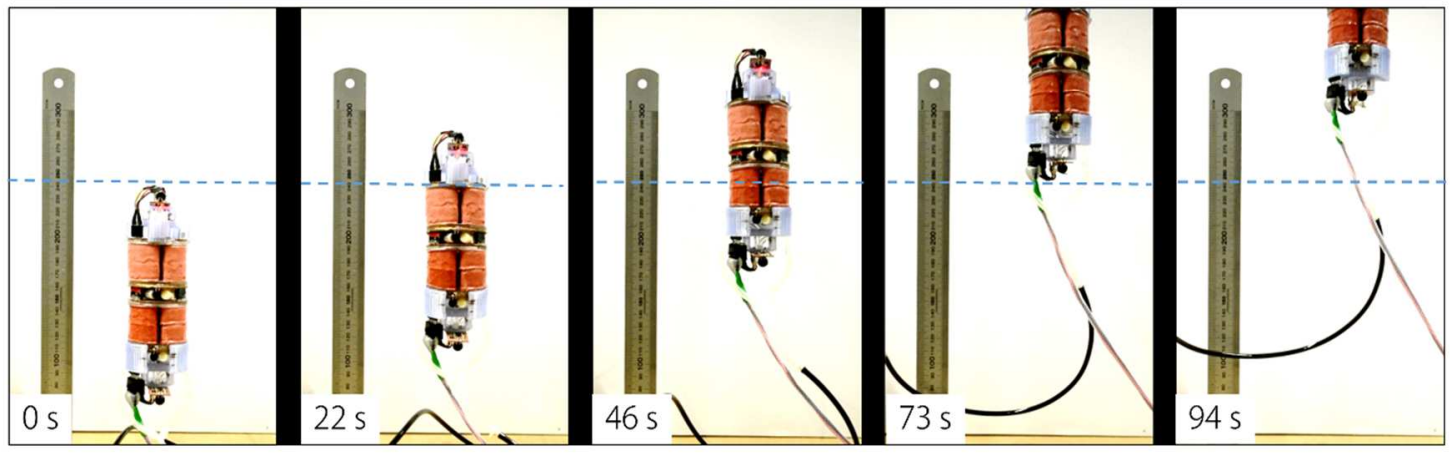

Fig. 5. Diverse locomotion modes of modular continuum robot. A series of frames captured from video is shown for each of four different gait modes: vertical climbing with suction cup modules (A), wave gait with 5 V-SPA Modules (B), wave gait with 3 V-SPA Modules $(\mathbf{C})$, and rolling gait (D).

\section{Interaction task: Multi-modal locomotion}

Alternate modes of locomotion were also found to be possible using the modular vacuum driven soft continuum robot system suitable for potential use in diverse and variable environments. Up to five V-SPA Modules were assembled in series and programmed to achieve two distinct gaits following strategies investigated previously for continuum style snake robots (45-47): a wave gait and a rolling gait. The first of these is shown with a sequence of video frames in Figure 5B depicting the progression of the forward wave gait over a flat, level surface with a measured $5 \mathrm{~mm} / \mathrm{s}$ average speed $(0.05 \mathrm{BL} / \mathrm{s})$. As a secondary but inherited feature of the modular robot structure, the reconfigurability and robustness of the continuum robot are also exhibited in Figure 5C where locomotion is preserved (and even improved in terms of average speed, measuring $11 \mathrm{~mm} / \mathrm{s}(0.08 \mathrm{BL} / \mathrm{s}))$ after two modules were removed from the robot, without requiring any changes to the gait controller. The increase in speed results only from an inherent change in vacuum supply air flow, which is restricted more for every module attached. Better designs could compensate for this by simply providing a central supply line with larger diameter.

Lateral motion was also accomplished utilizing a rolling gait, expanding the utility of the mobile robot to suit different locomotion objectives. This gait, shown in Figure 5D, is capable of much higher locomotion speeds $(60 \mathrm{~mm} / \mathrm{s}$ average speed or $1.33 \mathrm{BL} / \mathrm{s})$, than the wave gait of any robot length; however, this is possible only at the cost of a larger operational workspace (wider body span relative to the direction of travel). In practice the 
multi-modal functionality of this type of robot may be exploited to achieve fast motion over generally wide, open terrain using the rolling gait, while slower, cautious navigation through narrow passages, gaps, or obstructions could be approached using the wave gait, which operates within a more slender body width. A demonstration of the continuum robot gaits described can be found in the supplemental Movie S6.

\section{Mechanical tuning: Jamming-based stiffening}

While our initial workspace testing indicated the compliance of the arm does not significantly affect repeatability, manipulation testing of the soft arm, as well as experience from literature (48), reveals the controllability of such a soft structure to precise locations remains a challenge. This was apparent from qualitative observations of object manipulation/relocation tasks, where the object mass in addition to the inertia of the arm itself led to oscillatory behavior. While closing the active control loop to better maintain positioning and path following is likely needed for the best results, these tasks would benefit from other methods of "passive control", including the ability to tune mechanical system parameters of soft robotic devices themselves. In particular, this motivated the design and integration of a peripheral variable stiffness module, to provide the ability to change the overall rigidity or passivity of the soft arm.

Distributed across the length of the continuum robot arm, this module is comprised of multiple stiffening pillars in parallel with each actuator module, and utilizes jamming of enclosed granular media (ground coffee) driven by actively controlled vacuum to vary the stiffness of the overall structure. Figure 6 shows the resulting stiffness characteristics of the soft continuum robot fitted with the stiffening module, when force is applied through a high-strength nylon thread (fishing line) at the distal module perpendicular to the axis of the vertically mounted robot. Jamming pillars were spaced in three columns around each VSPA Module, and powered together with $88.5 \%$ vacuum when activated. The tested directions are grouped as either a pull in the direction of a jamming column, toward the vertex of the triangle formed from a top view of the three columns, or toward an edge, between two jamming columns. Five cycles were performed in each direction, with the result for each condition provided as an average of these trials. The measurements indicate an increase of stiffness in every direction when jamming is enabled, with the greatest increase always in the direction of a vertex, and hence the most direct compression of a single jamming column, with increases of $38.8 \%, 48.5 \%$, and $31.7 \%$ when vacuum is applied versus not. In the direction of an edge, the stiffness in was only moderately increased from their activation, by $15.1 \%, 31.5 \%$, and $17.3 \%$ for the three edge direction loading conditions. A diagram of the test setup is shown in Figure S2, along with a depiction of the fabrication process used to produce the granular jamming units. 


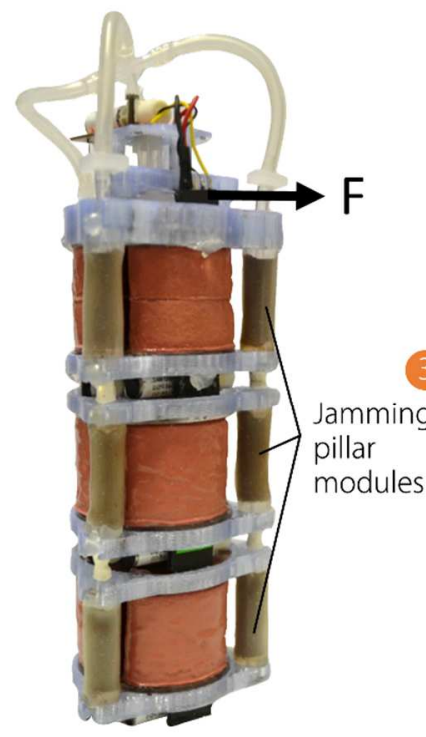

(A)

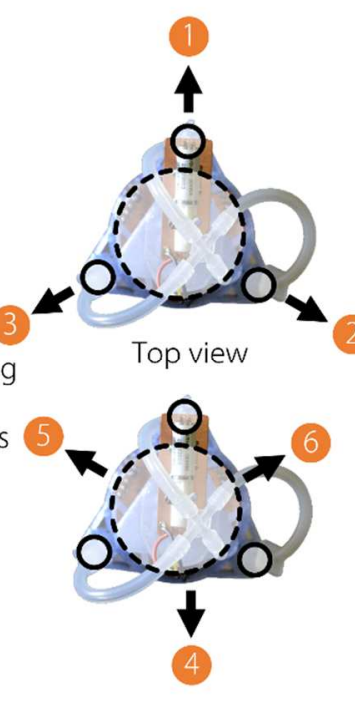

(B)

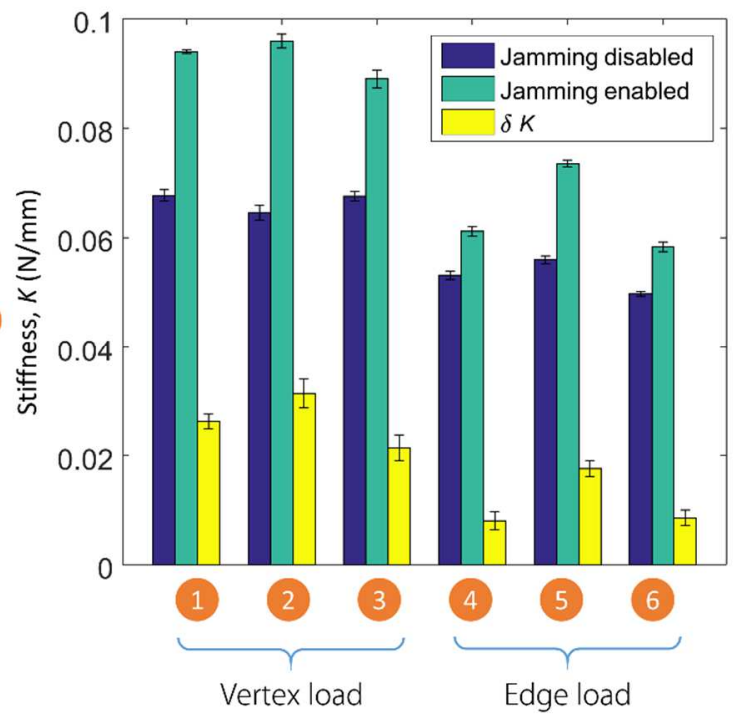

(C)

Fig. 6. Granular cellular matrix jamming enables active stiffness tuning of vacuum-driven soft structures. The addition of a jamming module enables stiffening of a continuum robot comprised of three $\mathrm{V}$ SPA Modules (A). The stiffness was measured using a vertical material testing machine, configured with a grounded pulley to redirect vertical motion of a cable to the horizontal direction. Tensile force and displacement measured in the vertical direction were thereby mapped to the direction perpendicular to the initially vertical axis of the actuator structure. The cable was attached at the top of the distal module, and the assembly was rotated in increments of $60^{\circ}$ to six total positions for measurements in multiple radial directions, with five loading cycles performed in each position $(\mathbf{B})$. The measured stiffness shown in $(\mathbf{C})$ increased in every direction when the jamming module was activated, in comparison to its inactive state. Error bars represent one standard deviation.

The jamming modules in this demonstration are coupled and joined to the same controlled vacuum input but could equally be included as part of more advanced actuator modules with internally integrated, independently controlled jamming pillars for more selective mechanical tuning capability of a soft structure. Additionally, only granular jamming was explored for this investigation of mechanical tuning, but stiffening with vacuum can also be achieved using flexible sheet materials sandwiched between air-sealed plastic film to achieve similar or possibly improved results $(30,49)$.

\section{Discussion}

The actuators presented here and the combined example applications illustrate the versatility of vacuum-based SPAs constructed from simple materials and methods. The fabrication procedure for V-SPAs reduces the time, tools, and cost required for creating actuators for soft robots by employing only 2D manufacturing methods and eliminating conventional molds, which allows rapid, batch production from minimal effort in comparison to other SPA types. Although conventional molding techniques inherently produce more uniform actuator structure and reduced variability between different actuators, the accuracy and repeatability of individual V-SPAs are not significantly affected as shown here, despite the non-homogenous wall thickness produced from manual application of silicone rubber layers. This improvement in actuator fabrication serves to decrease the design and development cycle time, leaving more room for focus on the system-level development of complete and useful soft robots. Toward the same goal, the employment of vacuum as a 
primary pneumatic supply source used to power V-SPAs enables more efficient integration of other vacuum-based mechanisms in a single soft robotic system allowing for more complex and diverse soft robot capability. This potential was effectively demonstrated through the use of modular units which allow many vacuum-driven mechanisms, as well as many redundant actuators to be seamlessly combined and tested in different soft robot configurations. Furthermore, unlike most other SPAs (6-16), utilizing a single source of pneumatic power significantly reduces the overhead cost of redundant subsystem infrastructure needed in comparison to the alternative case, where components, such as regulators, distribution manifolds, and central supply lines cannot be shared. This simplification saves weight and design effort.

V-SPAs likewise support the implementation of systems with many active DoF, through the use of lightweight foam material as a central component, helping to significantly reduce the weight-cost of additional actuation. In combination with plug-and-play architecture, this enables systems such as those demonstrated here with up to 15 actuators, or with fewer DoF and available payload capacity to support future integration of onboard batteries and power generation. Both of these aspects represent current goals and challenges of research in the soft robotics field, toward real-world application of the newly forming technologies coming from within it.

While the advantages of foam V-SPAs are promising for the development of a new type of complex soft robots, they are not without certain fundamental and practical limits. First of all, the capacity of vacuum to produce useful contractile work in an actuator is limited by the pressure of its external environment. For nominal use at standard atmospheric pressure this serves as an absolute limitation, however in more creative applications such as use in alternative environments (underwater or exo-planetary), this "limit" can be treated as a parameter to be considered in design. In any case, for a given setting this coupling to the environment imposes a boundary on the performance of the actuators, and hence the system as a whole. Beyond this inherent physical limit, the specific design implementation of VSPAs in soft robotic systems can also be seen as a limitation, as the actuators do not provide a consistent mechanical constraint alone to direct their motion unless used carefully. Although the 2D manufacturing method employed here can be adapted to allow for more complex shapes conducive to directed buckling (bellows-like shapes) and actuation, the most effective method for this is through the support of external constraints, such as reliance on the stiffness of an attached surface to restrict the buckling of the actuator along that interface, allowing it to occur only between two moving bodies as desired. Additionally, external structures can help impose constraints and define actuator motion, such as the stiffness of other coupled actuators or as a well-defined joint axis of rotation (see Movie S1). The rigid upper and lower plates, as well as the flexible but non-stretchable central conduit of the V-SPA Modules produced here serve these purposes in part, ensuring the contraction of the actuators results primarily in rotational motion.

The simplicity of this V-SPA concept encourages the development of even more creative techniques and applications for soft actuation. Other methods of elastic foam production could be employed in the future to create complex 3D structures, possibly deviating from the strict use of off-the-shelf materials. Variable stiffness skin components of either different thicknesses or material could be used to generate more complex actuation patterns, for increasingly advanced V-SPA designs using multi-material 3D printers or other standard soft robot fabrication tools. Regardless of the implementation method or application, future soft robots will benefit from exploring and exploiting the benefits of both foam based 
materials and vacuum power as new viable means to achieving versatile capabilities for environmental interaction as well as controlled modulation of mechanical system performance.

\section{Materials and Methods Objectives}

The objective of this study was to characterize and validate the performance and integration of V-SPAs in multifunctional vacuum-based soft robots. We explain the design procedure and fabrication techniques for the actuators and multi-DoF actuator modules, and describe test methods for obtaining performance metrics for each.

\section{Actuator design and fabrication}

The fabrication process of a V-SPA shown in Figure 7 begins with a basic preform structure, to act as a mount during application of the outer rubber layer and as a mask over the foam where connection of a vacuum supply to the internal actuator cavity is eventually made. For the actuators produced here the preform also served to create a smooth flat surface on the same side as the supply access mask when peeled off, so the actuator will form a good seal when glued to an equally smooth flat supply distribution plate.
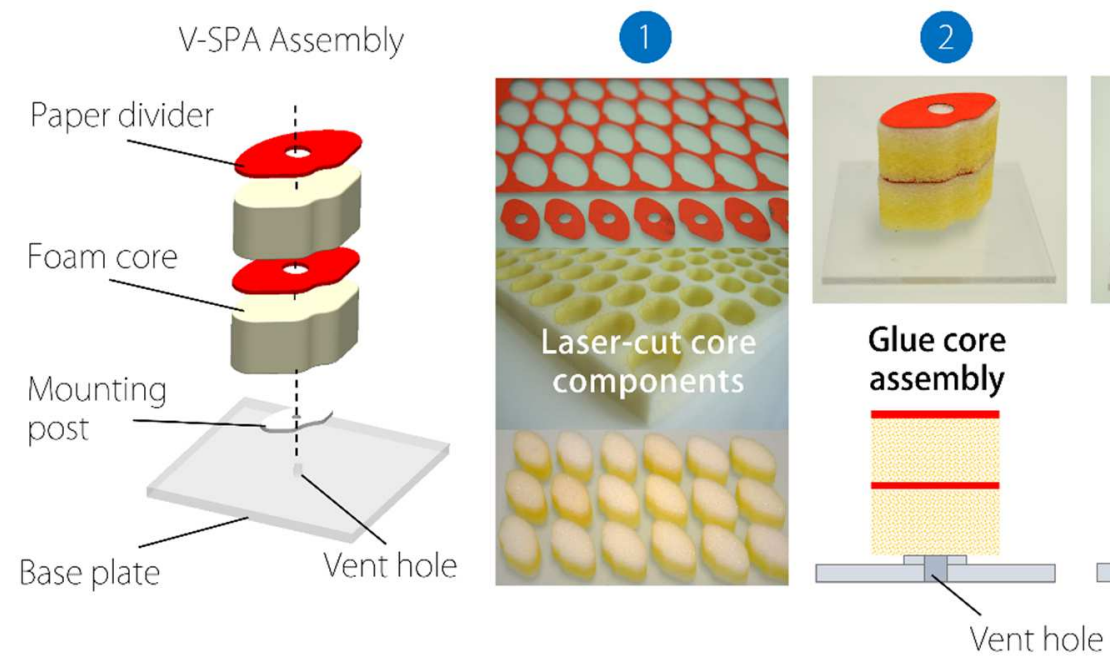

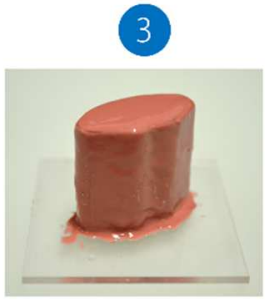

Brush-on silicone layers

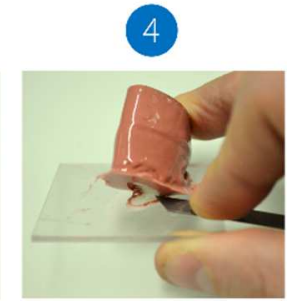

Ready for use
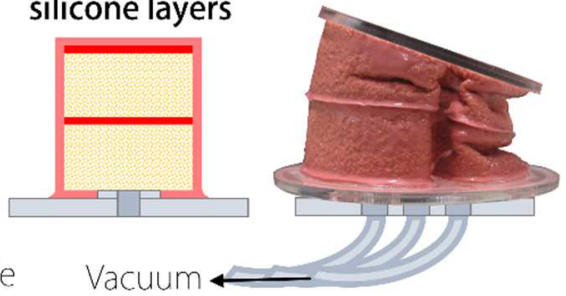

Fig. 7. Fabrication of V-SPAs. A four step process is shown for V-SPA fabrication. 1) Cut foam core shapes and paper divider plates using $\mathrm{CNC} \mathrm{CO}_{2}$ laser. 2) Assemble actuator cores and dividers on mounting post of preform structure of using cyanoacrylate gel. 3) Apply two coats of silicone rubber to outer surface of foam core assembly including the space between the bottom foam and baseplate. A vent hole is included through the mounting post and base plate to reduce the formation of bubbles caused by expanding internal air in the foam core when a heating oven is used to speed curing. Allow layer cure between coats. 4) Remove actuator from preform using a razor to separate the attached foam, or directly attach vacuum line to base plate through the vent hole.

The actuator itself is composed of 2D foam core shapes manufactured from a sheet of opencelled polyurethane foam (12.7 $\mathrm{mm}$ thickness polyurethane foam, McMaster-Carr, 8643K511) and rigid paper dividers. The porous foam cores serve as the main actuator body, while the paper dividers help reduce the buckling on the upper surface of the actuator, and at intervals along the height to enforce deformation primarily in the vertical direction. 
$\mathrm{A} \mathrm{CO}_{2}$ laser was used to cut many foam core and paper divider components from a single sheet at once, allowing batch production of the actuators. The foam cores and paper dividers are assembled in a stack with cyanoacrylate gel glue, and then glued to the preform. After preparation by mixing and degassing, two coats of ELASTOSIL ${ }^{\circledR}$ M 4601 silicone rubber are spread with brush-like strokes onto all exposed surfaces of the foam core using a flexible spreader (thin cardboard), while being cured at $50^{\circ} \mathrm{C}$ between coats. Once cured, the actuators can be removed from the preform and attached to a vacuum supply, or used directly on the preform by applying vacuum to the vent hole through the base.

While the basic construction of V-SPAs is described above, many variable parameters affect actuator performance. One of the leading design variables is geometry, as it pertains to dimensions which are easy to change, like actuator shape, and to others which are more difficult to manipulate, like the porosity of the foam used. As a starting point, we investigated only minor changes in actuator morphology toward a very specific goal, to create a circular module with 3 actuators and room at the center to pass wiring and a central pneumatic supply. This bounded the overall shape of the actuator to be used, but small improvements were added in an effort to achieve specific behaviors and subjectively improved performance criteria, in this case maximal linear deflection. To increase the directionality of the actuators, a particular geometry was selected for the foam core which would allow inward compression and buckling of sidewalls without interference limiting the downward actuator stroke. This helped to direct actuator contraction to the axial direction, perpendicular to the top plane of the original foam sheet. Secondly, multiple foam chambers were created simply by stacking individual foam cores to increase the actuator range-of-motion. To create independent porous volumes within a single actuator, thin 0.2 $\mathrm{mm}$ heavy-gauge paper dividers were assembled between two foam cores glued together in a stack to form an internal membrane. The dividers contain a $6 \mathrm{~mm}$ hole at the center to allow air flow between chambers while still enforcing separation. Without this membrane the overall structure of the two-core actuators would yield an effectively taller actuator which for the same given cross sectional area, would yield a more horizontal than vertical mode of collapse upon vacuum activation. While a parametric study was not conducted to properly determine an ideal $V$-SPA compression ratio, it is plausible an optimal relationship exists to best select actuator height based on cross-sectional geometry (aspect ratio) for linear actuation. For planar fabrication methods this ratio is the most accessible parameter to optimize, however an ideal actuator shape would incorporate geometric contours along the "walls" of the foam core to increase stress concentrations for uniform buckling, much in the same way a corrugated bellows collapses. This is partly achieved by creating multichamber actuators, whose stiff dividers act similarly to limit the stress along designated rings around the actuator perimeter. A separate study could investigate the optimal size and number of separate foam core chambers needed to maximize deflection or force using this design method.

Other factors including foam type and composition, porosity, and elastomer coating material are expected to greatly affect the performance of V-SPAs, but the methods for testing these variations involve greater effort. To begin, different foam materials and elastomers behave differently, and not all combinations allow for full curing of multi-part elastomers which chemically interact. Open-cell foam materials of different composition and porosity are also limited as off-the-shelf components, but can be produced from scratch for testing if desired. These factors limit the possibility for ready empirical parameterization testing. Perhaps a more controlled and available approach would seem to be simulating the effect and performance of actuator deformation using finite element modeling techniques, but this 
proves to be as difficult as it is circular, since accurate models of this type require empirically determined values for material properties. Nevertheless, as data and models improve over time, this technique will prove to be invaluable as actuators such as the VSPA are adopted for further use.

The use of primarily off-the-shelf materials allows this new type of actuator to be easily produced with low-resources, partly to extend the accessibility and scope of soft robot construction and research, especially toward the realm of lower education STEM (Science, Technology, Engineering, and Mathematics) outreach programs. The concept of foam vacuum actuation however is not exclusively tied to these materials and methods of manufacture. We do capitalize greatly from laser-cutting machines for fabricating 2D foam core shapes, but this tool is not required and virtually any method for cutting foam sheets will suffice. Early prototypes of V-SPAs were in fact built from scissor-cut foam cores attached to the end of wooden dowels (to serve as a mount and mask), and could be directly super-glued to a supply tube with the outer rubber skin used to seal the foam and fix the tube to the actuator in a single step. Alternative to brushing on the outer rubber layer, dipping might also be employed to coat an entire foam core for even faster manufacture time.

\section{V-SPA Module control and characterization}

Control of each V-SPA Modules connected in series is achieved over a single wire interface, enabled by a low cost and readily available integrated circuit (IC) originally designed for controlling individual LED pixels in networked displays (Worldsemi, WS2811). The IC is repurposed to supply an activation signal to onboard amplifiers for direct control over three embedded solenoid valves (Lee, LHDA0531115H) shadowing the operation of each color channel of an RGB (Red, Green, Blue) LED. A significant benefit of this IC is that it tolerates real time reconfiguration of hardware, enabling plug-and-play functionality of the V-SPA Modules. Robots can therefore be reduced or extended fundamentally to any number of modules on a single bus over relatively large spans, with only practical limitations imposed by RAM and electrical power required. Using standard microcontrollers, such as Arduino, hundreds of IC-enabled modular units can be controlled in a serial network through a single wire with little additional local electronics hardware, compared to alternative networking schemes (I2C, CAN, direct serial) which require more local computational power. Figure 4b depicts the topology of this network architecture, while a schematic of the internal electronic connections to the LED driver IC can be seen in the supplemental Figure S3.

To determine the performance characteristics of a V-SPA Module, a step response was empirically measured for multiple values across different conditions. Each response was averaged from three different actuators, cycled 10 times each. This process was repeated for 10 conditions of varying load, and 10 conditions of varying input vacuum. Load was applied by calibrated weights through a cable hung over a pulley and attached to a short level arm fixed to the module. Vacuum pressure was varied by changing the positive supply pressure to a vacuum ejector module (SMC ZH05B). The cycles for all three actuators in each condition were averaged to obtain a single representative step response curve from which performance metrics were extracted. The maximum angular motion is directly measured, while the rise time is found from the time between response crossings at $10 \%$ and $90 \%$ of the maximum angular deflection. The initial angular velocity is also found in this region, 
from a linear fit to the first half of the actuator motion (up to 50\%), where the response curve is dominantly linear.

Blocked moment measurements of the V-SPA Module were obtained using a six-axis force and torque sensor (Nano17 SI-25-0.25, ATI Industrial Automation) mounted to the upper plate of the module constrained at the base in a rigid test fixture. The reported value was averaged from individual activation of three different actuators, cycled 10 times each. Using an alternate setup, the average mechanical power output of a V-SPA Module was also estimated through measurements of displacement, $\Delta h$, and time, $\Delta t$, for a known mass, $m$ fixed to a cable hung over a pulley (assumed to be a constant force, $F=m g$ ), and driven by a short arm attached to the module, following $P=F \cdot \Delta h / \Delta t$. The metrics of torque and power were normalized by the mass of the module as well as the actuator alone to compute specific torque $\left(\tau_{s}\right)$, specific power $\left(P_{S}\right)$, and specific energy $\left(E_{S}\right)$. While the latter of these is relatively low compared to other vacuum powered actuators $(20)$, with $P_{s}=5 \mathrm{~W} \mathrm{~kg}^{-1}$ and $E_{s}=1.2 \mathrm{~J} \mathrm{~kg}^{-1}$, the value of specific torque is found to be relatively high, with $\tau_{s}=45.3 \mathrm{~N} \cdot \mathrm{m}$ $\mathrm{kg}^{-1}\left(45.3 \mathrm{~N} \cdot \mathrm{mm} \mathrm{g}^{-1}\right)$, more than five times that of the modular DC motor powered actuators designed for a conventional rigid continuum robot, the Unified Snake (50).

\section{List of Supplementary Materials}

Fig. S1. Anatomy of a V-SPA

Fig. S2. Fabrication and testing of jamming module

Fig. S3. Single wire module networking with serial LED driver IC.

Movie S1. Binary control of 3-DoF V-SPA array without modular interface

Movie S2. Binary workspace of V-SPA Module

Movie S3. Continuum robot repeatability test

Movie S4. Vacuum suction manipulation with continuum robot

Movie S5. Vacuum suction climbing with payload

Movie S6. Vacuum robot locomotion

\section{References}

1. R. Ikeura, H. Inooka, Variable impedance control of a robot for cooperation with a human, in Proceedings of 1995 IEEE International Conference on Robotics and Automation (1995), vol. 3, pp. 3097-3102 vol.3.

2. A. Calanca, R. Muradore, P. Fiorini, Impedance Control of Series Elastic Actuators Using Acceleration Feedback, in Wearable Robotics: Challenges and Trends (Springer, Cham, 2017), pp. 33-37.

3. R. Pfeifer, M. Lungarella, F. Iida, Self-Organization, Embodiment, and Biologically Inspired Robotics. Science. 318, 1088-1093 (2007).

4. R. Pfeifer, M. Lungarella, F. Iida, The challenges ahead for bio-inspired "soft" robotics. Commun. ACM. 55, 76 (2012).

5. C. Laschi, B. Mazzolai, M. Cianchetti, Soft robotics: Technologies and systems pushing the boundaries of robot abilities. Sci. Robot. 1, eaah3690 (2016). 
6. K. C. Galloway, P. Polygerinos, C. J. Walsh, R. J. Wood, Mechanically programmable bend radius for fiber-reinforced soft actuators, in 2013 16th International Conference on Advanced Robotics (ICAR) (2013), pp. 1-6.

7. M. A. Robertson, H. Sadeghi, J. M. Florez, J. Paik, Soft Pneumatic Actuator Fascicles for High Force and Reliability. Soft Robot. (2016), doi:10.1089/soro.2016.0029.

8. P. Moseley et al., Modeling, Design, and Development of Soft Pneumatic Actuators with Finite Element Method. Adv. Eng. Mater. 18, 978-988 (2016).

9. G. Agarwal, N. Besuchet, B. Audergon, J. Paik, Stretchable Materials for Robust Soft Actuators towards Assistive Wearable Devices. Sci. Rep. 6, 34224 (2016).

10. D. Rus, M. T. Tolley, Design, fabrication and control of soft robots. Nature. 521, 467-475 (2015).

11. A. D. Marchese, R. K. Katzschmann, D. Rus, A Recipe for Soft Fluidic Elastomer Robots. Soft Robot. 2, 7-25 (2015).

12. G. Krishnan, J. Bishop-Moser, C. Kim, S. Kota, Kinematics of a Generalized Class of Pneumatic Artificial Muscles. J. Mech. Robot. 7, 041014 (2015).

13. B. Tondu, Modelling of the McKibben artificial muscle: A review. J. Intell. Mater. Syst. Struct. 23, 225-253 (2012).

14. B. Mosadegh et al., Pneumatic Networks for Soft Robotics that Actuate Rapidly. Adv. Funct. Mater. 24, 2163-2170 (2014).

15. H. A. Sonar, J. Paik, Soft Pneumatic Actuator Skin with Piezoelectric Sensors for Vibrotactile Feedback. Front. Robot. AI. 2 (2016).

16. A. Firouzeh, M. Salerno, J. Paik, Soft pneumatic actuator with adjustable stiffness layers for Multi-DoF Actuation, in 2015 IEEE/RSJ International Conference on Intelligent Robots and Systems (IROS) (2015), pp. 1117-1124.

17. C. Eppner, R. Deimel, J. Álvarez-Ruiz, M. Maertens, O. Brock, Exploitation of environmental constraints in human and robotic grasping. Int. J. Robot. Res. 34, 1021-1038 (2015).

18. M. T. Tolley et al., A Resilient, Untethered Soft Robot. Soft Robot. 1, 213-223 (2014).

19. F. Ilievski, A. D. Mazzeo, R. F. Shepherd, X. Chen, G. M. Whitesides, Soft Robotics for Chemists. Angew. Chem. Int. Ed. 50, 1890-1895 (2011).

20. D. Yang et al., Buckling Pneumatic Linear Actuators Inspired by Muscle. Adv. Mater. Technol. 1, 1600055 (2016).

21. D. Yang, M. S. Verma, E. Lossner, D. Stothers, G. M. Whitesides, Negative-Pressure Soft Linear Actuator with a Mechanical Advantage. Adv. Mater. Technol. 2, 1600164 (2017).

22. B. C. Mac Murray et al., Poroelastic Foams for Simple Fabrication of Complex Soft Robots. Adv. Mater. 27, 6334-6340 (2015). 
23. A. Argiolas et al., Sculpting Soft Machines. Soft Robot. 3, 101-108 (2016).

24. I. M. Van Meerbeek et al., Morphing Metal and Elastomer Bicontinuous Foams for Reversible Stiffness, Shape Memory, and Self-Healing Soft Machines. Adv. Mater. 28, 2801-2806 (2016).

25. B. Trimmer, Soft Robots and Size. Soft Robot. 2, 49-50 (2015).

26. E. Brown et al., Universal robotic gripper based on the jamming of granular material. Proc. Natl. Acad. Sci. 107, 18809-18814 (2010).

27. F. Tramacere, M. Follador, N. M. Pugno, B. Mazzolai, Octopus-like suction cups: from natural to artificial solutions. Bioinspir. Biomim. 10, 035004 (2015).

28. Y. J. Kim, S. Cheng, S. Kim, K. Iagnemma, A Novel Layer Jamming Mechanism With Tunable Stiffness Capability for Minimally Invasive Surgery. IEEE Trans. Robot. 29, 10311042 (2013).

29. N. G. Cheng et al., Design and Analysis of a Robust, Low-cost, Highly Articulated manipulator enabled by jamming of granular media, in 2012 IEEE International Conference on Robotics and Automation (2012), pp. 4328-4333.

30. J. L. C. Santiago, I. D. Walker, I. S. Godage, Continuum robots for space applications based on layer-jamming scales with stiffening capability, in 2015 IEEE Aerospace Conference (2015), pp. 1-13.

31. M. Cianchetti et al., Soft Robotics Technologies to Address Shortcomings in Today's Minimally Invasive Surgery: The STIFF-FLOP Approach. Soft Robot. 1, 122-131 (2014).

32. W. McMahan et al., Field trials and testing of the OctArm continuum manipulator, in Proceedings 2006 IEEE International Conference on Robotics and Automation, 2006. ICRA 2006. (2006), pp. 2336-2341.

33. P. Qi et al., A Novel Continuum Manipulator Design Using Serially Connected DoubleLayer Planar Springs. IEEEASME Trans. Mechatron. 21, 1281-1292 (2016).

34. M. W. Hannan, I. D. Walker, The `elephant trunk' manipulator, design and implementation, in 2001 IEEE/ASME International Conference on Advanced Intelligent Mechatronics, 2001. Proceedings (2001), vol. 1, pp. 14-19 vol.1.

35. K. Suzumori, S. Iikura, H. Tanaka, Development of flexible microactuator and its applications to robotic mechanisms, in 1991 IEEE International Conference on Robotics and Automation Proceedings (1991), pp. 1622-1627 vol.2.

36. G. S. Chirikjian, thesis, California Institute of Technology (1992).

37. J. Burgner-Kahrs, D. C. Rucker, H. Choset, Continuum Robots for Medical Applications: A Survey. IEEE Trans. Robot. 31, 1261-1280 (2015).

38. H. Marvi et al., Sidewinding with minimal slip: snake and robot ascent of sandy slopes. Science. 346, 224-229 (2014). 
39. B. Bayat, A. Crespi, A. Ijspeert, Envirobot: A Bio-Inspired Environmental Monitoring Platform, in IEEE/OES Conference on Autonomous Underwater Vehicles (AUV) (Tokyo, Japan, 2016).

40. S. Hirose, Biologically Inspired Robots: Serpentile Locomotors and Manipulators (Oxford University Press, 1993).

41. A. A. Stokes, R. F. Shepherd, S. A. Morin, F. Ilievski, G. M. Whitesides, A Hybrid Combining Hard and Soft Robots. Soft Robot. 1, 70-74 (2013).

42. P. Liljebäck, Ø. Stavdahl, K. Y. Pettersen, Modular Pneumatic Snake Robot: 3D Modelling, Implementation And Control. Model. Identif. Control Nor. Res. Bull. 29, 21-28 (2008).

43. C. D. Onal, D. Rus, Autonomous undulatory serpentine locomotion utilizing body dynamics of a fluidic soft robot. Bioinspir. Biomim. 8, 026003 (2013).

44. G. Gerboni et al., Modular soft mechatronic manipulator for minimally invasive surgery (MIS): overall architecture and development of a fully integrated soft module. Meccanica. 50, 2865-2878 (2015).

45. J. Dai et al., Robot-inspired biology: The compound-wave control template, in 2015 IEEE International Conference on Robotics and Automation (ICRA) (2015), pp. 5879-5884.

46. K. Melo, L. Paez, Experimental determination of control parameter intervals for repeatable gaits in modular snake robots, in 2014 IEEE International Symposium on Safety, Security, and Rescue Robotics (2014) (2014), pp. 1-7.

47. M. Mori, S. Hirose, Three-dimensional serpentine motion and lateral rolling by active cord mechanism ACM-R3, in IEEE/RSJ International Conference on Intelligent Robots and Systems, 2002 (2002), vol. 1, pp. 829-834 vol.1.

48. M. Manti, V. Cacucciolo, M. Cianchetti, Stiffening in Soft Robotics: A Review of the State of the Art. IEEE Robot. Autom. Mag. 23, 93-106 (2016).

49. J. Ou et al., jamSheets: Thin Interfaces with Tunable Stiffness Enabled by Layer Jamming, in Proceedings of the 8th International Conference on Tangible, Embedded and Embodied Interaction (ACM, New York, NY, USA, 2013), TEI '14, pp. 65-72.

50. C. Wright et al., Design and architecture of the unified modular snake robot, in 2012 IEEE International Conference on Robotics and Automation (2012), pp. 4347-4354.

51. adafruit/Adafruit_NeoPixel. GitHub, (available at https://github.com/adafruit/Adafruit_NeoPixel). 


\section{Acknowledgments}

We thank Dr. Amy Wu, École Polytechnique Fédérale de Lausanne (EPFL), for assistance with motion capture and data processing, and Dr. Kamilo Melo, KM-RoBoTa, for counsel on the subject of snake robotics which greatly improved this manuscript. Funding: This work was supported by the Swiss National Science Foundation Fund for RoBuSt project (205321_163292), and the Swiss National Centre of Competence in Research (NCCR) Robotics. Author contributions: M.A.R. conceived the concepts, designed and built the hardware, designed and conducted experiments, analyzed data, and wrote the manuscript. J.P. Developed the concepts, directed the research, designed the experiments, and edited the manuscript. Competing interests: No competing financial interests exist. Data and materials availability: Contact J.P. for any questions regarding EAGLE CAD files, schematics, and parts list for electrical components, Solidworks CAD models for a single V-SPA Module assembly with dimensions as fabricated here, and for experimental raw data and processing scripts. 


\section{Supplementary Materials}

\section{Figures}

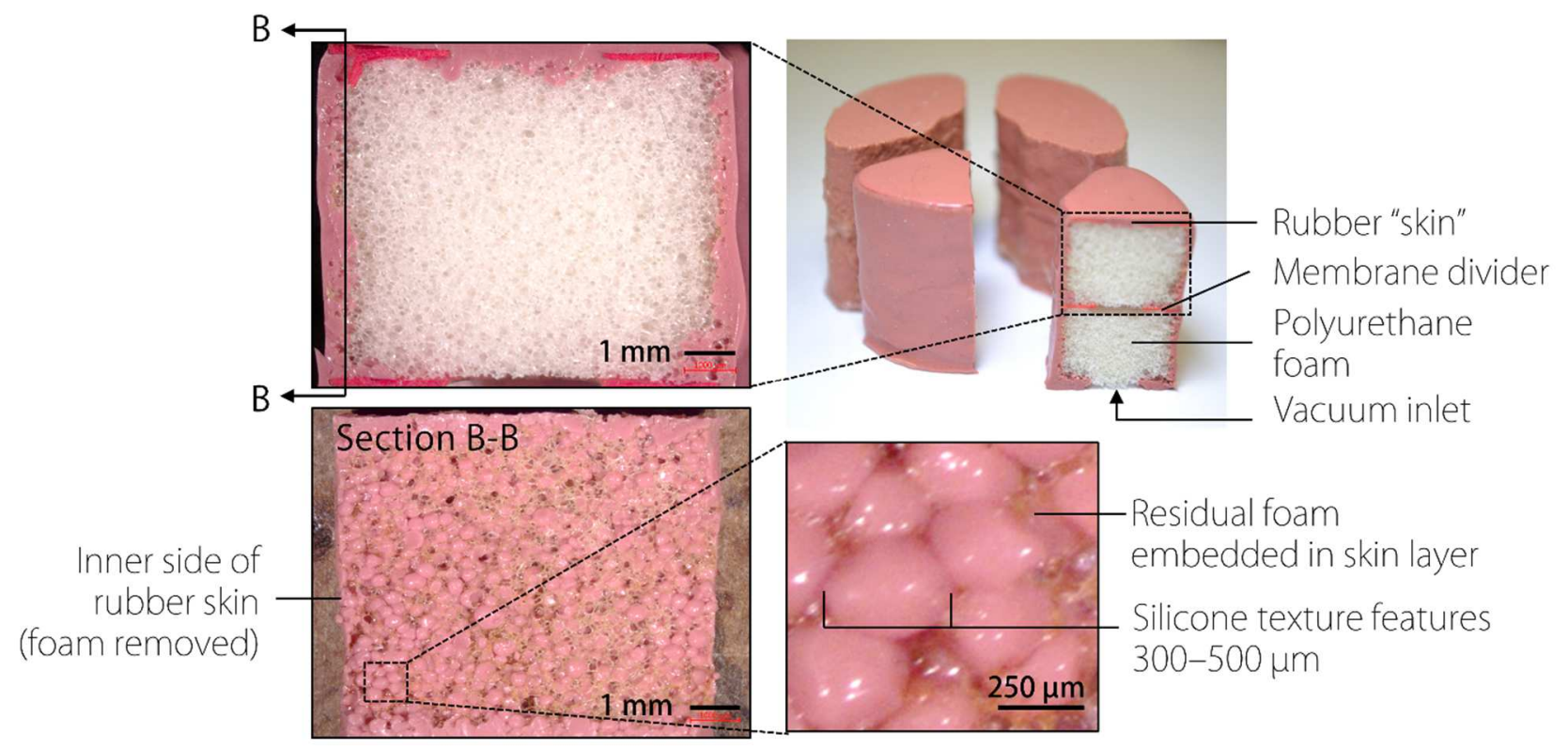

Fig. S1. Anatomy of a V-SPA. When uncured silicone is applied to the surface of the foam core it penetrates to a depth limited by its viscosity and liquid surface tension, and then remains primarily on the surface of the foam. The bubble-like texture features visible in the cross section are formed across the open pores of the foam core, while some foam remains embedded in the skin layer of the actuator. It can be seen that the wall thickness for V-SPAs is very non-uniform, however this does not significantly impede repeatability or robustness as the actuators perform well, with minimal variability, in testing over many cycles. 

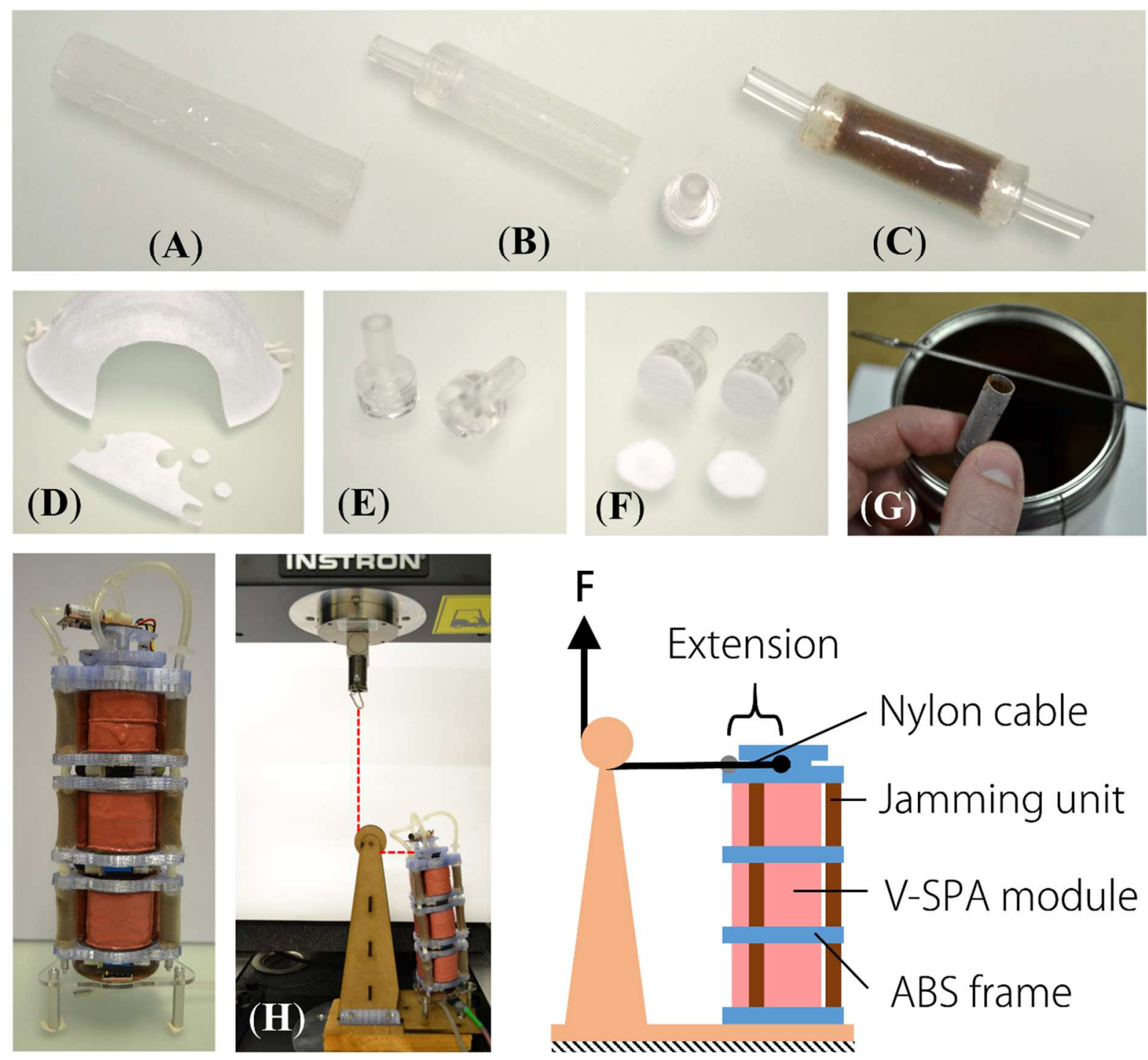

Fig. S2. Fabrication and testing of jamming module. Jamming units are fabricated by first creating thin walled silicone tubes using Dragon Skin-50 brushed onto a cylindrical form. The resulting cured silicone tube is peeled off the form and a segment is cut to make one jamming unit (A). End caps are attached to each end of the tube (B), after being filled with fine ground coffee particles $(\mathbf{C})$. To ensure the coffee is not drawn out of the module from the vacuum supply, disc shaped filters are cut from a paper dust mask (D). Before inserting into the silicone tube, the end caps are fitted with segments of vinyl tubing to attach to the vacuum supply $(\mathbf{E})$ and the paper filters are glued on the inside surface $(\mathbf{F})$. Filling with coffee is accomplished by first gluing one end cap in place to the silicone tube segment, scooping and packing in a measured amount of coffee to the open end, and then finally gluing the other end cap in place $(\mathbf{G})$. Photo $(\mathbf{H})$ depicts the actual test set up used to measure the stiffness of the stacked modules and jamming units with a dashed red line superimposed over the translucent nylon cable. The adjacent illustration shows the setup with an explanation of important components, as well as the parameters measured during the stiffness evaluation. 


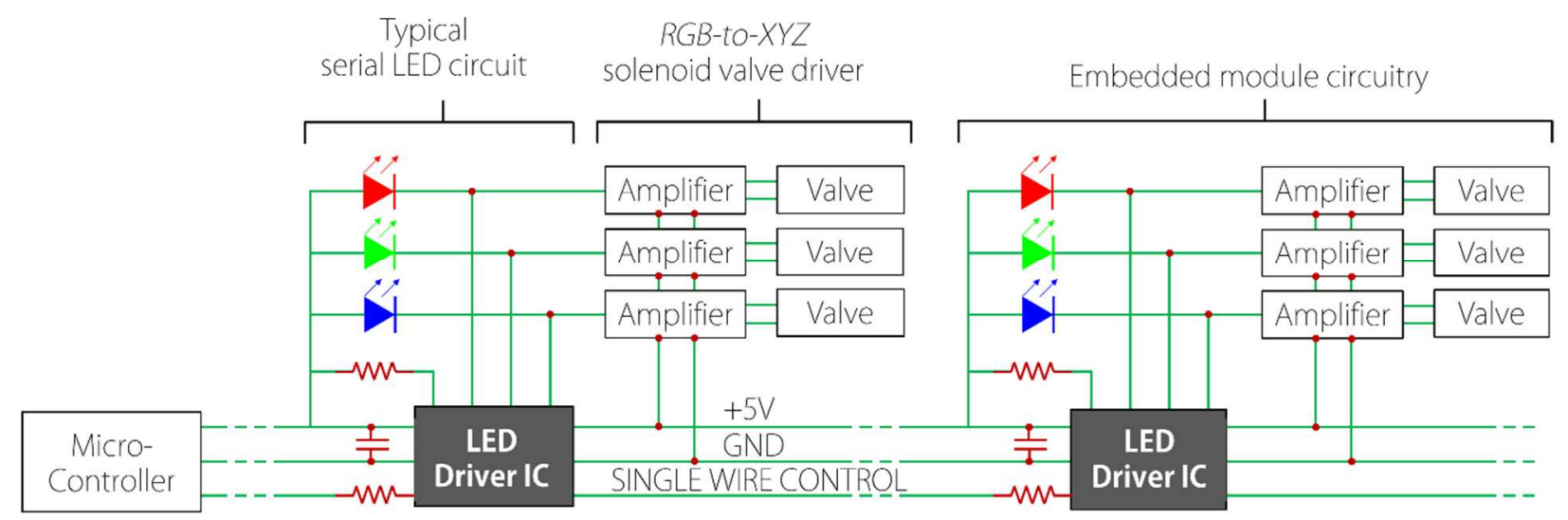

Fig. S3. Single wire module networking with serial LED driver IC. Circuitry embedded within each actuator module contains a three-channel IC designed and widely used for controlling multi-color Red-Green-Blue (RGB) LEDs. In parallel with the color channels, these chips can be leveraged to provide three-channel control of solenoid valves through an amplifier. Each channel is addressed over a single wire interface, using a time-based data packet structure which is passed to subsequent drivers connected in series. Communication libraries for Arduino based microcontrollers are available from Adafruit Industries on GitHub (51).

\section{Movie Captions}

Movie S1. Binary control of 3-DoF V-SPA array without modular interface. A circular array of V-SPAs are assembled on a distribution manifold with each actuator connected to a vacuum supply through a manually triggered solenoid valve. The actuators are first activated alone to illustrate the nominal mode of vacuum-induced buckling. The addition of a plate to the top surface of all actuators with Loctite 406 adhesive prevents buckling of the actuator top surface and the added constraint of stiffness from the other unactuated V-SPAs y an angular deflection of the upper plate. In addition to singular, binary activation of the V-SPAs, they are also activated in pairs to yield a total of 6 possible discrete angular positions of the upper plate, spaced at $60^{\circ}$ around the center of the array. Movie_S1.mp4

Movie S2. Binary workspace of V-SPA Module. A 3-DoF V-SPA Module demonstrates rapid angular deflection as shown in randomized directions. Discrete, binary control is utilized to operate each V-SPA individually to produce motion in three primary directions at $120^{\circ}$ spacing around the central axis of the actuator. While not shown here, finer, more continuous control of the direction of deflection or magnitude of the angle is possible with this module utilizing PWM vacuum pressure control. Movie_S2.mp4

Movie S3. Continuum robot repeatability test. A ten-cycle trial test was performed under an OptiTrack motion capture system, which accurately recorded the trajectory of the continuum robot mounted vertically. The repeatability of the robot was evaluated by analyzing the path of the robot endpoint, which in the video is shown to be a single, offset reflective marker. While the data set for the test shown in this video was not recorded properly, another test was conducted to produce the data shown in Figure 4, for which three markers were used to track the calculated center point of the distal module. Black tape was 
used to obscure other reflective components on the robot which interfere with the motion tracking system. The activation pattern that can be seen was selected deliberately as a progression from the most distal module to that most proximal to the base with a delay between each activation to limit the magnitude of oscillation caused by the actuator deflection, and not a direct consequence of the inflation or manifold dynamics. All actuators on a side can in fact be activated simultaneously, but the resulting torque from the sudden strong movement would result in longer waiting between subsequent cycles to allow dissipation of large oscillations. Movie_S3.mp4

Movie S4. Vacuum suction manipulation with continuum robot. A single active suction cup module is added to the distal end of a Vacuum-powered Continuum Robot comprised of three V-SPA Modules to serve as an end effector. A valve connected to the internal volume of the suction cup connects it to the central vacuum supply to enable selective adhesion or release of smooth, flat objects. The robot demonstrates a simple pick-and-place operation by moving empty acrylic containers from a gravity-fed supply rack to target bins on either side of the robot base. The motion of the robot is programmed open-loop and the adhesion of the particular suction cup used depends is sensitive to alignment, but could easily be replaced with a more compliant, auto-aligning variety designed more specifically for such operations. The suction cup used was adapted from a fixture system designed for long-term adhesion to smooth bathroom tiles. Movie_S4.mp4

Movie S5. Vacuum suction climbing with payload. The payload capacity of a climbing robot was estimated using increments of $10 \mathrm{~g}$ mass weights suspended from a nylon cable. The largest mass tolerated that did not inhibit vertical progress in climbing was found to be $70 \mathrm{~g}$. The next tested increment of $80 \mathrm{~g}$ was found to disrupt the climbing pattern of the robot, however this may result from the given controller used for the vertical gait and as a consequence of additional passive degrees of freedom at the lower "foot" which allows the robot to briefly fall away from the wall. Beyond this threshold, better timing (faster release of footholds and body actuation) may be used to prevent falling, although previous tests also found that this would likely yield slower climbing speeds. Movie_S5.mp4

Movie S6. Vacuum robot locomotion. Three gaits are demonstrated, in four configurations; a vertical climbing gait, with two actuator modules, and two additional suction cup modules achieves locomotion at $2 \mathrm{~mm} / \mathrm{s}$, or 0.01 Body Lengths per second (BL/s). A rolling gait and wave gait pattern are demonstrated with a 5 module, 15 DoF continuum robot, while the wave gait is repeated with a 3 module, 9 DoF robot. The shorter 3 module continuum robot achieves faster forward locomotion of $11 \mathrm{~mm} / \mathrm{s}$, than the 5 module version of $5 \mathrm{~mm} / \mathrm{s}$, while the rolling gait achieves the fastest average speed at $60 \mathrm{~mm} / \mathrm{s}$. None of the gaits tested were optimized for speed, but the variety is shown to illustrate the characteristic diversity of the different locomotion modes. The robots shown are powered from dual pneumatic ports at each end of the body to compensate for pressure and flow drop in undersized module ports, although both supplies are connected to a single, internal central supply line connected to every V-SPA in the robot through localized solenoid valves. Movie_S6.mp4

This is the author's version of the work. It is posted here by permission of the AAAS for personal use, not for redistribution. The definitive version was published in Science Robotics, Vol.2, 30 Aug, 2017, doi:

10.1126/scirobotics.aan6357 